\title{
Research on Spatial Co-Agglomeration Between Financial Services Industry and Manufacturing in Zhejiang Province
}

\author{
Chang-juan ZHENG ${ }^{1, a}$, Chao Zhang ${ }^{2, b^{*}}$ \\ ${ }^{1,2}$ Ningbo Finance and Economics University, Ningbo 315175,Zhejiang, China \\ azhengchangjuan@163.com, bacdzhangchao@163.com
}

Key words: financial services industry; manufacturing; Co-Agglomeration; Zhejiang

\begin{abstract}
Based on the actual data of county in Zhejiang province, this paper analyses the levels of agglomeration of financial services industry and co-agglomeration between financial services industry and manufacturing in Zhejiang province. The conclusions are as follows: The levels of financial services industry agglomeration is low in past and currently belonging to moderate agglomeration, and was inclined "N"-type upward trend; the levels of co-agglomeration are high between financial services industry and manufacturing. From the geographical point of view, northeastern of Zhejiang province was significantly higher than that in southwestern of Zhejiang province.
\end{abstract}

\section{Introduction}

During the $13^{\text {th }}$ Five-Year Plan period, Zhejiang, as an important province of China featuring manufacturing industry cluster, has been accelerating the agglomeration of financial services industry and strengthening the financial industry, thus to promote economic development, cultivate new economic growth point, and promote the transformation and upgrading of manufacturing industry. Therefore, this paper explores the spatial relationship between Zhejiang financial services and manufacturing industry and its internal development mechanism, as based on the perspective of industrial agglomeration, thus, we can have an understanding on the interactive relationship between these two major industry in Zhejiang, and discover the spatial agglomeration law of interactive industrial development.

In 1997, Ellison and Glaeser discovered that co-agglomeration exists among industries with upstream and downstream division of labor ${ }^{[1]}$. The subsequent researches were mainly carried out from two aspects: the common agglomeration measure of industry and the formation mechanism of common agglomeration ${ }^{[2-6]}$. At present, most researches take the agglomeration among industries as the object of study, and empirical research on the co-agglomeration of financial services industry and manufacturing industry is rather rare; a few studies on the co- agglomeration among secondary and tertiary industries, manufacturing industry and production services industry were based on the provincial or prefecture city level, and studies on county spatial level is rare. In view of this, the paper, by taking E-G modification indices for reference, analyzes the levels of agglomeration of financial services industry and manufacturing and co-agglomeration between these two in Zhejiang province, and makes attempt to explain its trend and formation mechanism.

\section{Research methods and data}

\subsection{Research methods}

Co-agglomeration index. Since the initiation of EG index, Ellision and Glaeser proposed the new EG modification index to measure the co-agglomeration level of industries. However, Devereux et al. noticed that the calculation of EG modification index is too complex, so they simplified the EG modification index as follows:

$$
C(r)=\left(G_{r}-\sum_{j=1}^{r} w_{j}^{2} G_{j}\right) /\left(1-\sum_{j=1}^{r} w_{j}^{2}\right)
$$

Of which: 


$$
w_{j}=T_{j} / \sum_{j=1}^{r} T_{j}
$$

refers to the ratio of employment quantity $T_{j}$ in the single industry $j$ in a specified region to the total employment quantity $T_{j}$ in all industries; $j$ refers to the number of industries; $G_{r}$ refers to the spatial agglomeration formed by industries; $G_{j}$ refers to the spatial agglomeration level formed by single industry, and is obtained through the calculation of Herfindahl index.

\subsection{Data}

The data used in this paper are from the 2003-2016 Statistical Yearbook of Zhejiang. The whole province is divided into two regions, the Northeast region and the Southwest region, thus to compare the industry agglomeration of Zhejiang by region. Northeast Zhejiang includes Hangzhou, Ningbo, Jiaxing, Huzhou, Shaoxing and Zhoushan, while Southeast Zhejiang includes Wenzhou, Jinhua, Quzhou, Taizhou and Lishui.

\section{Agglomeration level and evolution trend of financial services industry}

\subsection{Based on regional perspective}

The overall spatial agglomeration of the financial services industry in the earlier stage is at a low level but moderate in the current, and was inclined " $N$ "-type upward tread . Based on EG index, in the past 14 years since 2002, the general agglomeration of the financial services industry has varied towards "N"-type trend. From 2002 to 2007, EG index increased from 0.001661 at the lowest to 0.007531, which however has generally decreased from 2007 to 2011. Then from 2011 on, EG index has increased continuously, which has broken through 0.02 between 2013 and 2015 and reached the peak value in 2015 .

\subsection{Based on local perspective}

$\mathrm{G}$ index of financial industry of the northeastern part of Zhejiang province is increasing year by year while $\mathrm{H}$ index is decreasing, and besides, EG index of the financial industry in northeastern Zhejiang is consistently growing and has been maintained around 0.03 at the end, reflecting a moderate agglomeration level; as similar to northeastern Zhejiang, the evolution trend of G index in southwest of Zhejiang is also growing upward while $\mathrm{H}$ index is decreasing and EG index is increasing upward, which has maintained around 0.06 finally, reflecting a high agglomeration level.

\section{Co-agglomeration characteristics of financial services industry and manufacturing industry}

\subsection{Based on regional perspective}

This papers adopts modified EG index to measure the co-agglomeration characteristics between the financial services and manufacturing industries. The results has showed us the overall variation situation on the co-agglomeration degrees of financial services industry and manufacturing industry of Zhejiang province from 2002 to 2015. Since 2002, both co-agglomeration levels of financial services and manufacturing industries of Zhejiang have maintained above 0.02 , which is the critical value of moderate agglomeration, and except for the 4 years between 2003 and 2006 when the co-agglomeration was at a moderate level, in the rest years, the co-agglomeration of the two industries was at a high level and has reached the peak value at 0.063856 in 2014 . This shows there is a distinct co-agglomeration between the financial services and manufacturing industries in Zhejiang and it has basically maintained at a high agglomeration level.

\subsection{Based on local perspective}

Although the co-agglomeration between financial and manufacturing industries of Zhejiang is high, seeing from the inside of the region, due to the different economic growth basis, industrial structures and regional characteristics between northeastern and southwestern Zhejiang, the 
industrial co-agglomerations of these two places are also somehow different. The results show that although both co-agglomerations of financial and manufacturing industries in northeastern and southwestern Zhejiang are above moderate levels, the co-agglomeration level of northeastern Zhejiang between 2002 and 2015 was higher that of southwestern Zhejiang.

And then, this paper has used formula (2-1) to calculate and rank the co-agglomeration levels of the 11 prefecture-level cities of Zhejiang (see Table 1) and it's not difficult for us to find that all the co-agglomeration levels of these 11 prefecture-level cities are above 0.05 . Specifically speaking, the co-agglomeration levels of the two industries in cities of northeastern Zhejiang rank the first class, including Zhoushan, Hangzhou and Ningbo, however the co-agglomeration levels of the rest cities are lower than Quzhou and Taizhou in southwestern Zhejiang, especially Jiaxing and Shaoxing that are relatively backward. This shows that although generally the co-agglomeration between financial services and manufacturing industries in northeastern Zhejiang is higher than that in southwestern Zhejiang, there are still distinct regional imbalance internally.

Table 1. Ranking on Co-agglomeration Levels of 11 Prefectural-level Cities of Zhejiang

\begin{tabular}{cccc}
\hline Region & Prefecture-level city & Co-agglomeration level & Ranking \\
\hline & Hangzhou & 0.59 & 2 \\
& Ningbo & 0.42 & 3 \\
\multirow{3}{*}{ Northeastern Zhejiang } & Jiaxing & 0.05 & 11 \\
& Huzhou & 0.22 & 6 \\
& Shaoxing & 0.07 & 9 \\
& Zhoushan & 0.62 & 1 \\
\hline \multirow{5}{*}{ Southwestern Zhejiang } & Wenzhou & 0.15 & 7 \\
& Jinhua & 0.06 & 4 \\
& Quzhou & 0.29 & 5 \\
& Taizhou & 0.28 & 8 \\
\hline
\end{tabular}

\section{Conclusions and suggestions}

(1)The overall spatial agglomeration of the financial services industry in the earlier stage is at a low level but moderate in the current, and was inclined " $\mathrm{N}$ "-type upward tread; the agglomeration level of the financial industry of southwestern Zhejiang is higher than that of northeaster Zhejiang.

(2)There is a distinct co-agglomeration between the financial services and manufacturing industries in Zhejiang and it has basically maintained at a high agglomeration level, of which, the co-agglomeration between financial services and manufacturing industries in northeastern and southwestern Zhejiang is above moderate level and although generally the co-agglomeration level in northeastern Zhejiang is higher than that in southwestern Zhejiang, there are still distinct regional imbalance internally.

(3)As two economically leading prefecture-level cities, the co-agglomeration levels of Hangzhou and Ningbo are second to none.

In the next step, Zhejiang shall grasp every opportunity to optimize the industrial distribution and actively guide on the interactive development between financial services industry and manufacturing industry. Government departments at all levels shall highly concentrate on financial supportive points, financial growth poles, financial centers and various levels of financial agglomeration zone construction and in compliance with the spatial layout of financial industry "one zone one feature" take this financial agglomeration zone construction work as an important measure to promote the relative industrial development and co-agglomeration between financial services and manufacturing industries. 


\section{Acknowledgement}

This work was supported by Zhejiang Provincial Philosophy and Social Science Planning Project (Grant No. 17NDJC105YB), the foundation of Zhejiang Science Bureau (Grant No. LY17D010007), the foundation of Ningbo Science Bureau (2016A10003), the foundation of Zhejiang Federation of Social Sciences(Grant No. 2018Z24) and Cixi Social Science Project (Grant No.2017SKB001)

\section{References}

[1] Glenn E, Edwar G. Geographic Concentration in U. S. Manufacturing Industrial clusters : A Dartboard Approach. Journal of Political Economy, 105(5): 889-927, 1997.

[2] Barrios S, Bertinelli L, Strobl E. Coagglomeration and spillovers. Regional Science \& Urban Economics, 36(4):467-481, 2006.

[3] Rusche K, Kies U, Schulte A. Measuring spatial co-agglomeration patterns by extending ESDA techniques. Review of Regional Research, 31(1):11-25, 2011.

[4] Lu Jiangyong \& Tao Zhigang. Industrial Agglomeration and Co-agglomeration in China's Manufacturing Industries: with International Comparison. Economic Research, 2006(3):103-114.

[5] Zheng Wei. Spatial Agglomeration Level Measurement and Evaluation of Manufacturing in Fujian Province. Economic Geography, 32(7):74-80, 2012.

[6] He Junqing, Cao Mingming \& Wang Yanlin. Analysis on Spatial Evolution and Effect of Urban Industrial Agglomeration of Guanzhong - Based on Manufacturing Industry. Human Geography, 131(3):96-100, 2013. 